\title{
Massive Vector Particles Tunneling From Noncommutative Charged Black Holes and its GUP-corrected Thermodynamics
}

\author{
Ali Övgün \\ Physics Department, Eastern Mediterranean University, \\ Famagusta, Northern Cyprus, via Mersin 10, Turkey \\ Kimet Jusufi \\ Physics Department, State University of Tetovo, Ilinden Street nn, 1200, Macedonia
}

(Dated: September 25, 2018)

\begin{abstract}
In this paper, we investigate the tunneling process of charged massive bosons $W^{ \pm}$(spin-1 particles) from noncommutative charged black holes such as charged RN black holes and charged BTZ black holes. By applying the WKB approximation and by using the Hamilton-Jacobi equation we derive the tunneling rate and the corresponding Hawking temperature for those black holes configuration. Furthermore, we show the quantum gravity effects using the GUP on the Hawking temperature for the noncommutative $\mathrm{RN}$ black holes. The tunneling rate shows that the radiation deviates from pure thermality and is consistent with an underlying unitary theory.

PACS numbers: $04.62 .+\mathrm{v} ; 04.70$. Dy;11.30.-j

Keywords: Hawking radiation, Hawking temperature, Proca equation, Vector particles tunneling
\end{abstract}

\section{INTRODUCTION}

Hawking's amazing discovery that black holes should radiate thermally, undoubtedly, remains as one of the most interesting results in physics. Basically, Hawking used semiclassical arguments and showed that black holes must radiate thermally just as a black body [1]. Furthermore, due to the thermal nature of this radiation the initial pure state is mapped to a final mixed state in a non-unitary process which leads to the famous information loss paradox.

Over the years, people were able to derive the Hawking temperature by applying different techniques and methods 2]. Among others, the quantum tunneling has attracted a great interest. The so called null geodesic approach, introduced and developed by Kraus-Wilczek-Parkih [3 [8] and the Hamilton-Jacobi approach proposed and developed by Angheben-Padmanabhan [9 11]. A great number of different black hole configurations have been studied in details showing that the Hawking temperature does not depend on the particular coordinate transformation we use and the nature of the particles being emitted from black holes. In particular, the tunneling of scalar particles [12, 13], spin-1/2 particles [14 17], spin-3/2 [18, 19] and spin-1 particles [20 26], have been successfully investigated. Furthermore, recently quantum gravity effects of the entangled particles tunneling is investigated by using the generalized uncertainty principle [27]. The calculations shows that the tunneling rate deviates from pure thermality and is consistent with unitary theory.

On the other hand, since the discovery of Banados-Teitelboim-Zanelli (BTZ) black hole[47], three dimensional black holes become popular research area of quantum gravity. Today, the three dimensional gravity is used to test the theories behind the AdS/CFT correspondence. In Ref. [49], the authors analyzed the gravitational Aharonov-Bohm effect due to BTZ black hole in a noncommutative background. Moreover, in Ref. [50], has been analyzed the behavior of a particle test in the noncommutative BTZ space-time. The thermodynamic properties of the charged BTZ black hole were investigated in Ref. [51].

Recently, noncommutative gravity has attracted a lot of interest. Several black hole solutions have been found by using a new model of commutativity, including the neutral and charged black holes [28, 29], inspired higherdimensional charged black holes [30], and rotating charged black holes [31]. Furthermore, it is shown that this model of noncommutativity preserves the Lorentz invariance, Unitarity and UV-finiteness of quantum field theory 32 34]. According to this scenario, the black hole evaporation process might finish when the black hole reaches some minimal mass $M_{0}$, this on the other hand might shed some light on the information loss paradox. The key idea in noncommutative gravity is the noncommutativity of the spacetime points. This noncomutativity can be encoded in

\footnotetext{
*Electronic address: ali.ovgun@emu.edu.tr

${ }^{\dagger}$ Electronic address: kimet.jusufi@unite.edu.mk
} 
terms of noncommutative self-adjoint operators given by the commutator

$$
\left[x^{\mu}, x^{\nu}\right]=i \theta^{\mu \nu}
$$

where $\theta^{\mu \nu}$ is an anti-symmetric second-rank tensor. One natural consequence of this noncommutativity is the minimum length in spacetime. It should be noted that the point matter/charge distribution should be expressed by a Gaussian function rather than a Dirac delta function. The quantum tunneling of Hawking radiation also has been investigated in context of noncommutative black holes [35-38]. As we know from particle physics, a massive boson is a spin-1 particle described by the Proca equation. In particular, the charged massive bosons i.e., $W^{ \pm}$and $Z$ are very important in the Standard Model of particle physics, they are known as a force carriers of the weak interaction and for the important role in the confirmed Higgs Boson. In this context it's interesting to note the possible existence of a massive photon or the so-called Darklight [55, 56], and the charged spin-1 boson speculated as a potential dark matter candidates [57, 58]. On the other hand, due to the nontrivial interaction between the charged massive boson ( $W^{ \pm}$) fields and the electromagnetic field may lead to new insights. Therefore, we think the study of spin-1 particles merits more attention, in particular, in the context of black hole physics.

During the last few years, modification of the uncertainty principle to a generalized uncertainty principle (GUP) becomes important tool to investigate the effect of the quantum gravity on many different research areas 42, 5254]. Divergences in physics is suggested that it can be solved easily by using GUP relation instead of the using of usual Heisenberg uncertainty relation. Recently in Ref.([43]), the authors study the corrections for the entropy of the noncommutative BTZ black holes. In this paper, using the same procedure we investigate the GUP-corrected thermodynamics of charged black holes.

Inspired by what has just been said, in this work we follow [44], and study the tunneling of charged massive bosons $\left(W^{ \pm}\right)$from noncommutative charged black holes. The field equations can be derived by using the Lagrangian given by the Glasgow-Weinberg-Salam model. We then use the WKB approximation and the separation of variables which results with a set of four linear equations. Solving for the radial part by using the determinant of the metric equals zero, we found the tunneling rate and the corresponding Hawking temperature. Finally, we aim to compute the corrected Hawking temperature and entropy by incorporating the noncommutative parameter $\theta$ and quantum gravity effects $\alpha$.

The paper is organized as follows. In Sec. II, we investigate the tunneling of massive vector particles from the noncommutative charged Reissner-Nordström (RN) black hole and calculate the corresponding Hawking radiation. In Sec. III, we extend the tunneling of massive vector particles from noncommutative BTZ black holes and compute the corresponding Hawking temperature. In Sec. IV, we study the GUP-corrected Hawking temperature then we compute the GUP-Entropy corrections for the noncommutative charged RN black hole. Finally, in Sec. V, we comment on our results.

\section{TUNNELING FROM NONCOMMUTATIVE RN BLACK HOLES}

In noncommutative gravity the mass and charge distributions cannot be localized as point like particles but rather diffused and therefore given by a Gaussian distribution of some minimal width $\sqrt{\theta}$, as follows

$$
\rho_{\text {matt. }}(r)=\frac{M}{(4 \pi \theta)^{3 / 2}} e^{-\frac{r^{2}}{4 \theta}}
$$

respectively,

$$
\rho_{e l .}(r)=\frac{Q}{(4 \pi \theta)^{3 / 2}} e^{-\frac{r^{2}}{4 \theta}} .
$$

Here, the parameter $\theta$ encodes the minimal length due to the noncommutativity of the spacetime points. The line element which solves the Einstein's field equation in noncommutative spacetime is given by [29]

$$
d s^{2}=-f(r) d t^{2}+f(r)^{-1} d r^{2}+r^{2}\left(d \theta^{2}+\sin ^{2} \theta d \varphi^{2}\right)
$$

where

$$
f(r)=\left(1-\frac{2 M_{\theta}}{r}+\frac{Q_{\theta}^{2}}{r^{2}}\right)
$$


Using (2) and (3), the smeared mass and charge distributions can be expressed in terms of the lower incomplete Gamma functions as follows

$$
\begin{aligned}
M_{\theta} & =\frac{2 M}{\sqrt{\pi}} \gamma\left(\frac{3}{2}, \frac{r^{2}}{4 \theta}\right), \\
Q_{\theta} & =\frac{Q}{\sqrt{\pi}} \sqrt{\gamma^{2}\left(\frac{1}{2}, \frac{r^{2}}{4 \theta}\right)-\frac{r}{\sqrt{2 \theta}} \gamma\left(\frac{1}{2}, \frac{r^{2}}{2 \theta}\right)+r \sqrt{\frac{2}{\theta}} \gamma\left(\frac{3}{2}, \frac{r^{2}}{4 \theta}\right)}, \\
\gamma\left(\frac{a}{b}, u\right) & =\int_{0}^{x} \frac{d u}{u} u^{a / b} e^{-u} .
\end{aligned}
$$

Clearly, in the limit $\theta \rightarrow 0$, the noncommutative mass and charge solution reduces to the commutative case $M_{\theta} \rightarrow M$ and similarly $Q_{\theta} \rightarrow Q$. Solving for $f\left(r=r_{\theta}\right)=0$, we end up with the outer and inner horizons given by

$$
r_{\theta \pm}=M_{\theta \pm} \pm \sqrt{M_{\theta \pm}^{2}-Q_{\theta \pm}^{2}}
$$

Now, recall that the Lagrangian density, which describes the $W$-bosons in a background electromagnetic field can be written as 44

$$
\mathcal{L}=-\frac{1}{2}\left(D_{\mu}^{+} W_{\nu}^{+}-D_{\nu}^{+} W_{\mu}^{+}\right)\left(D^{-\mu} W^{-\nu}-D^{-\nu} W^{-\mu}\right)+\frac{m_{W}^{2}}{\hbar^{2}} W_{\mu}^{+} W^{-\mu}-\frac{i}{\hbar} e F^{\mu \nu} W_{\mu}^{+} W_{\nu}^{-},
$$

where $D_{ \pm \mu}=\nabla_{\mu} \pm \frac{i}{\hbar} e A_{\mu}$. Here $\nabla_{\mu}$ is the covariant derivative, $e$ gives the charge of the $W^{+}$boson, and $A_{\mu}$ is the electromagnetic potential of the black hole with components $A_{\mu}=\left(A_{0}, 0,0,0\right)$. Using the above Lagrangian the equation of motion for the $W$-boson field reads

$$
\frac{1}{\sqrt{-g}} \partial_{\mu}\left[\sqrt{-g}\left(D^{ \pm \nu} W^{ \pm \mu}-D^{ \pm \mu} W^{ \pm \nu}\right)\right] \pm \frac{i e A_{\mu}}{\hbar}\left(D^{ \pm \nu} W^{ \pm \mu}-D^{ \pm \mu} W^{ \pm \nu}\right)+\frac{m_{W}^{2}}{\hbar^{2}} W^{ \pm \nu} \pm \frac{i}{\hbar} e F^{\nu \mu} W_{\mu}^{ \pm}=0
$$

where $F^{\mu \nu}=\nabla^{\mu} A^{\nu}-\nabla^{\nu} A^{\mu}$. In this paper we will study the tunneling of $W^{+}$boson, therefore we need to solve the following equation

$$
\begin{array}{r}
\frac{1}{\sqrt{-g}} \partial_{\mu}\left[\sqrt{-g} g^{\mu \beta} g^{\nu \alpha}\left(\partial_{\alpha} W_{\beta}^{+}-\partial_{\beta} W_{\alpha}^{+}+\frac{i}{\hbar} e A_{\alpha} W_{\beta}^{+}-\frac{i}{\hbar} e A_{\beta} W_{\alpha}^{+}\right)\right] \\
+\frac{i e A_{\mu} g^{\mu \beta} g^{\nu \alpha}}{\hbar}\left(\partial_{\alpha} W_{\beta}^{+}-\partial_{\beta} W_{\alpha}^{+}+\frac{i}{\hbar} e A_{\alpha} W_{\beta}^{+}-\frac{i}{\hbar} e A_{\beta} W_{\alpha}^{+}\right)+\frac{m_{W}^{2} g^{\nu \beta}}{\hbar^{2}} W_{\beta}^{+}+\frac{i}{\hbar} e F^{\nu \alpha} W_{\alpha}^{+}=0
\end{array}
$$

for $\nu=0,1,2,3$. Using the WKB approximation

$$
W_{\mu}^{+}(t, r, \theta, \varphi)=C_{\mu}(t, r, \theta, \varphi) \exp \left(\frac{i}{\hbar} S(t, r, \theta, \varphi)\right)
$$

and taking the lowest order of $\hbar$, we end up with a set of four equations:

$$
\begin{aligned}
0 & =C_{0}\left(-\left(\partial_{1} S\right)^{2}-\frac{\left(\partial_{2} S\right)^{2}}{r^{2} f}-\frac{\left(\partial_{3} S\right)^{2}}{r^{2} f \sin ^{2} \theta}-\frac{m^{2}}{f}\right)+C_{1}\left(\left(\partial_{1} S\right)\left(e A_{0}+\partial_{0} S\right)\right)+C_{2}\left(\frac{\left(\partial_{2} S\right)}{r^{2} f}\left(\partial_{0} S+e A_{0}\right)\right) \\
& +C_{3}\left(\frac{\left(\partial_{3} S\right)}{r^{2} f \sin ^{2} \theta}\left(\partial_{0} S+e A_{0}\right)\right) \\
0 & =C_{0}\left(-\left(\partial_{1} S\right)\left(e A_{0}+\partial_{0} S\right)\right)+C_{1}\left(-f \frac{\left(\partial_{2} S\right)^{2}}{r^{2}}-f \frac{\left(\partial_{3} S\right)^{2}}{r^{2} \sin ^{2} \theta}+\left(\partial_{0} S+e A_{0}\right)^{2}-m^{2} f\right)+C_{2}\left(f \frac{\partial_{1} S \partial_{2} S}{r^{2}}\right) \\
& +C_{3}\left(f \frac{\partial_{1} S \partial_{3} S}{r^{2} \sin ^{2} \theta}\right), \\
0 & =C_{0}\left(-\partial_{2} S\left(\frac{\partial_{0} S+e A_{0}}{f}\right)\right)+C_{1}\left(f\left(\partial_{2} S\right)\left(\partial_{1} S\right)\right)+C_{2}\left(-f\left(\partial_{1} S\right)^{2}-\frac{\left(\partial_{3} S\right)^{2}}{r^{2} \sin ^{2} \theta}+\frac{\left(\partial_{0} S+e A_{0}\right)^{2}}{f}-m^{2}\right) \\
& +C_{3}\left(\frac{\left(\partial_{2} S\right)\left(\partial_{3} S\right)}{r^{2} \sin ^{2} \theta}\right),
\end{aligned}
$$




$$
\begin{aligned}
0 & =C_{0}\left(-\partial_{3} S\left(\frac{\partial_{0} S+e A_{0}}{f}\right)\right)+C_{1}\left(f\left(\partial_{3} S\right)\left(\partial_{1} S\right)\right)+C_{3}\left(-f\left(\partial_{1} S\right)^{2}-\frac{\left(\partial_{2} S\right)^{2}}{r^{2}}+\frac{\left(\partial_{0} S+e A_{0}\right)^{2}}{f}-m^{2}\right) \\
& +C_{2}\left(\frac{\partial_{2} S \partial_{3} S}{r^{2}}\right) .
\end{aligned}
$$

Due to spherical symmetry, one can choose $\theta=\pi / 2$, the non-zero elements of the coefficient matrix $\Xi$ are given by

$$
\begin{aligned}
& \Xi_{11}=\left(-\left(\partial_{1} S\right)^{2}-\frac{\left(\partial_{2} S\right)^{2}}{r^{2} f}-\frac{\left(\partial_{3} S\right)^{2}}{r^{2} f}-\frac{m^{2}}{f}\right) \\
& \Xi_{12}=-\Xi_{21}=\left(\partial_{1} S\right)\left(e A_{0}+\partial_{0} S\right) \\
& \Xi_{13}=\left(\frac{\left(\partial_{2} S\right)}{r^{2} f}\left(\partial_{0} S+e A_{0}\right)\right) \\
& \Xi_{14}=\left(\frac{\left(\partial_{3} S\right)}{r^{2} f}\left(\partial_{0} S+e A_{0}\right)\right) \\
& \Xi_{22}=\left(-f \frac{\left(\partial_{2} S\right)^{2}}{r^{2}}-f \frac{\left(\partial_{3} S\right)^{2}}{r^{2}}+\left(\partial_{0} S+e A_{0}\right)^{2}-m^{2} f\right) \\
& \Xi_{23}=f \frac{\left(\partial_{1} S\right)\left(\partial_{2} S\right)}{r^{2}} \\
& \Xi_{24}=f \frac{\left(\partial_{1} S\right)\left(\partial_{3} S\right)}{r^{2}} \\
& \Xi_{31}=-\frac{\left(\partial_{0} S+e A_{0}\right) \partial_{2} S}{f} \\
& \Xi_{32}=f\left(\partial_{2} S\right)\left(\partial_{1} S\right) \\
& \Xi_{33}=\left(-f\left(\partial_{1} S\right)^{2}-\frac{\left(\partial_{3} S\right)^{2}}{r^{2}}+\frac{\left(\partial_{0} S+e A_{0}\right)^{2}}{f}-m^{2}\right) \\
& \Xi_{34}=\Xi_{43}=\frac{\left(\partial_{2} S\right)\left(\partial_{3} S\right)}{r^{2}} \\
& \Xi_{41}=-\frac{\partial_{3} S\left(\partial_{0} S+e A_{0}\right)}{f} \\
& \Xi_{42}=f\left(\partial_{3} S\right)\left(\partial_{1} S\right) \\
& \Xi_{44}=\left(-f\left(\partial_{1} S\right)^{2}-\frac{\left(\partial_{2} S\right)^{2}}{r^{2}}+\frac{\left(\partial_{0} S+e A_{0}\right)^{2}}{f}-m^{2}\right) .
\end{aligned}
$$

Using the space-time symmetries of the metric (4), we can now choose the following ansatz

$$
S=-E t+W(r)+j \varphi+H(\theta)+C,
$$

and the nontrivial solution of this equation 20]

$$
\Xi\left(C_{0}, C_{1}, C_{2}, C_{3}\right)^{T}=0,
$$

is obtained by using the the determinant of the matrix $\Xi$ equals zero as given

$$
\left(f^{2} W^{\prime 2} r^{2}+\left(r^{2} m^{2}+\left(\partial_{2} H\right)^{2}\right) f-r^{2}\left(E-e A_{0}\right)^{2}\right)^{3} m^{2}=0
$$

Solving this equation for the radial part leads to the following integral

$$
W_{ \pm}= \pm \int d r \frac{1}{f} \sqrt{E^{2}-2 E e A_{0}+e^{2} A_{0}^{2}-f\left(m^{2}+\frac{\left(\partial_{2} H\right)^{2}}{r^{2}}\right)}
$$

Now, we can expand the function $f(r)$ in Taylor's series near the horizon

$$
f\left(r_{\theta+}\right) \approx f^{\prime}\left(r_{\theta+}\right)\left(r-r_{\theta+}\right),
$$


and by integrating around the pole at the outer horizon $r_{\theta+}$, gives [9, 10]

$$
W_{ \pm}= \pm \frac{i \pi \sqrt{E^{2}-2 E e A_{0}+e^{2} A_{0}^{2}}}{\left.f^{\prime}\right|_{r_{\theta+}}}
$$

Since every outside particle falls into the black hole with a $100 \%$ chance of entering the black hole , therefore, the corresponding probability of the ingoing massive vector particle must be set

$$
P_{-} \simeq e^{-2 \operatorname{Im} W_{-}}=1
$$

which implies also that

$$
I m S_{-}=I m W_{-}+\operatorname{Im} C=0
$$

On the other hand, for the outgoing particle we have

$$
I m S_{+}=I m W_{+}+\operatorname{Im} C,
$$

but we know that $\operatorname{ImC}=-\operatorname{Im} W_{-}$, and also from the Eq.(24) we see that

$$
W_{+}=-W_{-} .
$$

Therefore, the probability for the outgoing vector massive particle is given by

$$
P_{+}=e^{-2 I m S} \simeq e^{-4 I m W_{+/-}}
$$

Finally, the tunneling rate of massive vector particles tunneling from inside to outside the horizon is given by

$$
\Gamma=\frac{P_{+}}{P_{-}} \simeq e^{\left(-4 I m W_{+}\right)}=e^{-\frac{E_{n e t}}{T_{H}}}
$$

where $E_{n e t}=E-e A_{0}$. Then, the Hawking temperature of the noncommutative Reissner-Nordström black holes is recovered as

$$
T_{H}=\frac{1}{4 \pi} \frac{d f\left(r_{\theta+}\right)}{d r}
$$

Using Eq.(5), one can recover the Hawking temperature for noncommutative charged black hole, which is in agreement with [29, 35, 45]

$$
T_{H}=\frac{1}{4 \pi r_{\theta+}}\left[1-\frac{r_{\theta+}^{3} \exp \left(-\frac{r_{\theta+}^{2}}{4 \theta+}\right)}{4 \theta^{3 / 2} \gamma\left(\frac{3}{2}, \frac{r_{\theta+}^{2}}{4 \theta}\right)}\right]-\frac{Q^{2}}{\pi^{2} r_{\theta+}^{3}}\left[\gamma^{2}\left(\frac{3}{2}, \frac{r_{\theta+}^{2}}{4 \theta}\right)+\frac{r_{\theta+}^{3} \exp \left(-\frac{r_{\theta+}^{2}}{4 \theta}\right)\left(\gamma^{2}\left(\frac{1}{2}, \frac{r_{\theta+}^{2}}{4 \theta}\right)-\frac{r_{\theta+}}{\sqrt{2 \theta}} \gamma\left(\frac{1}{2}, \frac{r_{\theta+}^{2}}{2 \theta}\right)\right)}{16 \theta^{3 / 2} \gamma\left(\frac{3}{2}, \frac{r_{\theta+}^{2}}{4 \theta}\right)}\right]
$$

Therefore, one can recovers the classical Hawking temperature $T_{H}=\frac{\sqrt{M^{2}-Q^{2}}}{2 \pi\left(M+\sqrt{M^{2}-Q^{2}}\right)^{2}}$ on the commutative case. The Eq. (32) shows that in the noncommutative framework, there is not completely evaporation of the black hole inasmuch as it produces a Planck-sized remnant including the information. So information might be preserved in this remnant. The simple $r_{ \pm}$dependent form of the Hawking temperature from the noncommutative RN black hole can be approximately written as follows $T_{H}=\frac{\kappa(M, Q)}{2 \pi} \simeq \frac{1}{4 \pi} \frac{r_{\theta+}-r_{\theta-}}{r_{\theta+}^{2}}$, where the surface gravity $\kappa(M, Q)$ is $\frac{r_{\theta+}-r_{\theta-}}{2 r_{\theta+}^{2}}$.

\section{TUNNELING FROM CHARGED NONCOMMUTATIVE BTZ BLACK HOLES}

The line element describing the BTZ black hole in a noncommutative background given by [46, 49]:

$$
d s^{2}=-F^{2} d t^{2}+H^{-2} d r^{2}+2 r^{2} N^{\phi} d t d \phi+\left(r^{2}-\frac{\theta B}{2}\right) d \phi^{2}
$$


where the metric components are

$$
\begin{aligned}
F^{2} & =\frac{r^{2}-r_{+}^{2}-r_{-}^{2}}{l^{2}}-\frac{\theta B}{2} \\
H^{2} & =\frac{1}{r^{2} l^{2}}\left[\left(r^{2}-r_{+}^{2}\right)\left(r^{2}-r_{-}^{2}\right)-\frac{\theta B}{2}\left(2 r^{2}-r_{+}^{2}-r_{-}^{2}\right)\right], \\
N^{\phi} & =-\frac{r_{+} r_{-}}{l^{2} r^{2}} .
\end{aligned}
$$

where $B$ and $\theta$ are the magnitude of the magnetic field, and the noncommutative parameter, respectively. Furthermore, $r_{+}$and $r_{-}$are the outer and inner horizons. The event horizons are given as

$$
r_{ \pm}^{2}=\frac{l^{2} M}{2}\left[1 \pm \sqrt{1-\left(\frac{J}{M l}\right)^{2}}\right] .
$$

The metric given in Eq.(33) can be rewritten as

$$
d s^{2}=-f d t^{2}+Q^{-1} d r^{2}+\frac{J}{r} r d r d t+\left(1-\frac{\theta B}{2 r^{2}}\right) r^{2} d \phi^{2},
$$

where

$$
\begin{aligned}
& f=-M+\frac{r^{2}}{l^{2}}-\frac{\theta B}{2}, \\
& Q=-M+\frac{r^{2}}{l^{2}}+\frac{J^{2}}{4 r^{2}}-\frac{\theta B}{2}\left(\frac{2}{l^{2}}-\frac{M}{r^{2}}\right) .
\end{aligned}
$$

Here $\mathrm{M}$ is the mass, $\mathrm{J}$ is the angular spin of the BTZ black hole. Noted that the BTZ black hole without electric charge can be obtained as the quotient of AdS space. The vector potential of the black hole is given by

$$
A_{\mu}=\left(A_{t}, 0,0\right)
$$

where $A_{t}=-Q \ln \left(\frac{r}{l}\right)$.

To use the WKB approximation, as similar way done in section 2, we insert the wave function given in Eq.(13) and the vector potential into the Proca equation of motion for the $W$-boson field of Eq. (12) for the near the event horizon of a noncommutative BTZ black hole in the condition of $J=0$ and the metric is given as follows

$$
d s^{2}=-\tilde{f} d t^{2}+\tilde{Q}^{-1} d r^{2}+\left(r^{2}-K\right) d \phi^{2},
$$

where $K=\frac{\theta B}{2}, \tilde{f}=f^{\prime}\left(\hat{r}_{+}\right)\left(r-\hat{r}_{+}\right)$and $\tilde{Q}=Q^{\prime}\left(\hat{r}_{+}\right)\left(r-\hat{r}_{+}\right)$. Dividing by the exponential term and considering the leading terms yield three equations ;

$$
\begin{gathered}
0=\left[\frac{Q\left(r^{2}-K\right)\left(\partial_{r} S\right)^{2}+\left(\partial_{\phi} S\right)^{2}+m^{2}\left(r^{2}-K\right)}{f\left(r^{2}-K\right)}\right] C_{0}+\left[\frac{-Q\left[\left(\partial_{t} S\right)+A_{0} e\right] \partial_{r} S}{f}\right] C_{1} \\
+\left[\frac{-\left(\partial_{\phi} S\right)\left(\left(\partial_{t} S\right)+e A_{0}\right)}{f\left(r^{2}-K\right)}\right] C_{2}, \\
0=\left[-\frac{\left(\partial_{r} S\right)\left(\partial_{t} S+e A_{0}\right)}{f}\right] C_{0}+\left[\frac{\left(\partial_{\phi} S\right)\left(\partial_{r} S\right)}{r^{2}-K}\right] C_{2}+\left[\frac{\left(r^{2}-K\right)\left(\partial_{t} S+e A_{0}\right)^{2}-f\left(\partial_{\phi} S\right)^{2}-m^{2} f\left(r^{2}-K\right)}{f\left(r^{2}-K\right)}\right] C_{1} \\
0=\frac{\left[\left(\partial_{\phi} S\right)\left(\left(\partial_{t} S\right)+e A_{0}\right)\right]}{f} C_{0}+\left[-Q\left(\partial_{\phi} S\right)\left(\partial_{r} S\right)\right] C_{1}+\left[Q\left(\partial_{r} S\right)^{2}-\frac{\left(\left(\partial_{t} S\right)+e A_{0}\right)^{2}+m^{2} f}{f}\right] C_{2} .
\end{gathered}
$$

Using the spacetime symmetries one may choose the following ansatz

$$
S=-E t+W(r)+j \varphi+c,
$$


which leads to the following non-zero elements of the coefficient matrix $\mathbb{Z}$ are given by

$$
\begin{aligned}
& \mathbb{Z}_{11}=\left(\frac{Q W^{\prime 2}+m^{2}}{f}\right), \\
& \mathbb{Z}_{12}=-\frac{Q W^{\prime}}{f}\left(e A_{0}-E\right) \\
& \mathbb{Z}_{21}=-\frac{W^{\prime}}{f}\left(e A_{0}-E\right) \\
& \mathbb{Z}_{22}=\frac{\left(e A_{0}-E\right)^{2}-m^{2} f}{f} \\
& \mathbb{Z}_{33}=\frac{Q f W^{\prime 2}-\left(e A_{0}-E\right)^{2}+m^{2} f}{f} .
\end{aligned}
$$

Using the space-time symmetries of the metric Eq.(42), we can and the nontrivial solution of this equation

$$
\mathbb{Z}\left(C_{0}, C_{1}, C_{2}\right)^{T}=0,
$$

is obtained by using the the determinant of the matrix $\mathbb{Z}$ equals zero as given

$$
m^{2}\left(e^{2} A_{0}^{2}-2 e E A_{0}+E^{2}-f\left(m^{2}+Q f W^{\prime 2}\right)\right)^{2}=0 .
$$

Solving this equation for the radial part leads to the following integral

$$
W_{ \pm}= \pm \int d r \frac{1}{\sqrt{Q f}} \sqrt{E^{2}-2 E e A_{0}+e^{2} A_{0}^{2}-m^{2} f} .
$$

Solving this integral by integrating around the pole, gives

$$
W_{ \pm}= \pm \frac{i \pi \sqrt{E^{2}-2 E e A_{0}+e^{2} A_{0}^{2}}}{\left.\sqrt{Q^{\prime} f^{\prime}}\right|_{\hat{r}_{+}}} .
$$

By using similar arguments as in the last section, for the tunneling rate we must have

$$
\Gamma \simeq e^{\left(-4 \operatorname{Im} W_{+}\right)}=e^{-\frac{E_{n e t}}{T_{H}}},
$$

where $E_{n e t}=E-e A_{0}$.

In this way, Hawking temperature at the event horizon of the charged noncommutative BTZ black holes is calculated as

$$
T_{H}=\frac{1}{4 \pi} \sqrt{f^{\prime}\left(\hat{r}_{+}\right) Q^{\prime}\left(\hat{r}_{+}\right)}=\frac{\hat{r}_{+}}{2 \pi l^{2}}\left(1-\frac{\theta B r_{+}^{2}}{4 \hat{r}_{+}^{4}}\right)+\ldots
$$

Finally, the last result in terms of $r_{+}$, gives

$$
T_{H}=\frac{r_{+}}{2 \pi l^{2}}\left(1-\frac{\theta^{2} B^{2}}{16 r_{+}^{4}}\right)+\ldots
$$

Thus, we have recovered the Hawking temperature with remnant for the charged noncommutative BTZ black hole [46]. As expected, there are corrections for the Hawking temperature due to the noncommutativity of spacetime.

\section{GUP-ENTROPY AND CORRECTED HAWKING TEMPERATURE OF THE NONCOMMUTATIVE $R N$ BLACK HOLES}

In this section, by using the recent works in $([43,[52,53])$, we show the effect of the GUP to the Noncommutative $R N$ thermodynamics. Firstly, the GUP expression with a quadratic term in momentum is written as 54 ]

$$
\Delta x \Delta p \geq \hbar\left(1-\beta \Delta p+\beta^{2}(\Delta p)^{2}\right),
$$


where the dimensionless constant $\beta\left(\beta=\alpha l_{p} / \hbar\right)$, here $\alpha$ is a positive constant, $l_{p}$ and $\hbar$, are the Planck length $\left(l_{p}=\sqrt{\hbar G / c^{3}} \approx 10^{-35} \mathrm{~m}\right)$ and the Planck constant respectively. After some algebraic manipulation the last equation reduces to

$$
\Delta p \geq \frac{\hbar\left(\Delta x+\alpha l_{p}\right)}{2 \alpha^{2} l_{p}^{2}}\left(1-\sqrt{1-\frac{4 \alpha^{2} l_{p}^{2}}{\left(\Delta x+\alpha l_{p}\right)^{2}}}\right) .
$$

Note that $l_{p} / \Delta x$ is infinitesimally small compared with unity, one can therefore expand the last equation in Taylor series as follows

$$
\Delta p \geq \frac{1}{2 \Delta x}\left[1-\frac{\alpha}{2 \Delta x}+\frac{\alpha^{2}}{2(\Delta x)^{2}}+\cdots\right] .
$$

It is noted that after using the constant as a one $\left(G=c=k_{B}=1, \hbar=1\right.$, and $\left.l_{p}=1\right)$, uncertainty principle reduces to $\Delta x \Delta p \geq 1$. The saturated form of the Heisenberg uncertainty principle depended on energy of a particle can be also written in the form of $E \Delta x \geq 1$. Consequently, the new form of the Eq.(60) is

$$
E_{G U P} \geq E\left[1-\frac{\alpha}{2(\Delta x)}+\frac{\alpha^{2}}{2(\Delta x)^{2}}+\cdots\right],
$$

where $E_{G U P}$ is the corrected energy of the particle. Now we can rewrite the tunneling probability which we find in Eq.(30), by considering the effect of GUP as follows

$$
\Gamma \simeq \exp [-2 \operatorname{Im}(\mathcal{S})]=\exp \left[\frac{-2 \pi \mathrm{E}_{\mathrm{GUP}}}{\kappa}\right],
$$

where $\kappa$ is the surface gravity of noncommutative $R N$ black hole horizon given by $\kappa=2 f^{\prime}\left(r_{\theta_{+}}\right)$. Comparing this with the Boltzmann factor the GUP-corrected black hole temperature of noncommutative $R N$ is found as

$$
T_{G U P}=T_{H}\left[1-\frac{\alpha}{2(\Delta x)}+\frac{\alpha^{2}}{2(\Delta x)^{2}}+\cdots\right]^{-1},
$$

where $\Delta x=2 r_{\theta_{+}}=2\left(M_{\theta_{+}}+\sqrt{M_{\theta_{+}}^{2}-Q_{\theta_{+}}^{2}}\right)$. Thus, the corrected Hawking temperature due to the GUP-effects, reads

$$
T_{G U P}=\left(\frac{1}{4 \pi r_{\theta+}}[A]-\frac{4 Q^{2}}{\pi r_{\theta+}^{3}}[B]\right) \times\left(1+\frac{\alpha}{4 r_{\theta_{+}}}-\frac{\alpha^{2}}{8 r_{\theta_{+}}^{2}}+\cdots\right)
$$

where

$$
A=1-\left[\frac{r_{\theta+}^{3} \exp \left(-\frac{r_{\theta+}^{2}}{4 \theta+}\right)}{4 \theta^{3 / 2} \gamma\left(\frac{3}{2}, \frac{r_{\theta+}^{2}}{4 \theta}\right)}\right]
$$

and

$$
B=\gamma^{2}\left(\frac{3}{2}, \frac{r_{\theta+}^{2}}{4 \theta}\right)+\frac{r_{\theta+}^{3} \exp \left(-\frac{r_{\theta+}^{2}}{4 \theta}\right)\left(\gamma^{2}\left(\frac{1}{2}, \frac{r_{\theta+}^{2}}{4 \theta}\right)-\frac{r_{\theta+}}{\sqrt{2 \theta}} \gamma\left(\frac{1}{2}, \frac{r_{\theta+}^{2}}{2 \theta}\right)\right)}{16 \theta^{3 / 2} \gamma\left(\frac{3}{2}, \frac{r_{\theta+}^{2}}{4 \theta}\right)}
$$

It is noted that for $\alpha=0$, it is obtained the noncommutative $R N$ black hole temperature in Eq. (32). Therefore, we get more remnants for the noncommutative $R N$ black hole. Now let us compute the GUP-entropy corrections, for this reason we can now use the first law of $\mathrm{BH}$ thermodynamics and let for simplicity choose $\Delta x=2 r_{\theta_{+}}$. It follows

$$
\begin{aligned}
S_{G U P} & =\int \frac{d E}{T_{G U P}}=\int \frac{\kappa d A_{h}}{8 \pi T_{G U P}} \\
& =\int 2 \pi r_{\theta_{+}} d r_{\theta_{+}}\left[1-\frac{\alpha}{4 r_{\theta_{+}}}+\frac{\alpha^{2}}{8 r_{\theta_{+}}^{2}}+\cdots\right], \\
& =\pi r_{\theta_{+}}^{2}-\frac{\alpha \pi}{2} r_{\theta_{+}}+\frac{\alpha^{2} \pi}{4} \ln r_{\theta_{+}}+\cdots
\end{aligned}
$$


in the first and second line of the last equation we have used $\kappa=2 \pi T_{H}$ and $d A_{h}=8 \pi r_{\theta_{+}} d r_{\theta_{+}}$, respectively. Finally, we can rewrite the last equation in terms of the surface area of the event horizon as follows

$$
S_{G U P}=\frac{A_{h}}{4}-\frac{\alpha}{4} \sqrt{A_{h} \pi}+\frac{\pi \alpha^{2}}{8} \ln \frac{A_{h}}{4}+\cdots
$$

Notice that for $\alpha_{G U P}=0$, we recover the well-known area law for the BH mechanics i.e., $\left.S_{G U P}\right|_{\alpha=0} \rightarrow S=A_{h} / 4$. Remnants mainly due to extraordinary large amount of entropy confined within a tiny volume.

Note that in order to write explicitly the entropy in terms of $Q, M$ and the parameter $\theta$, we need to recall the following relation:

$$
r_{\theta_{+}}=M_{\theta_{+}}+\sqrt{M_{\theta_{+}}^{2}-Q_{\theta_{+}}^{2}},
$$

where

$$
\begin{aligned}
& M_{\theta_{+}}=M\left[\epsilon\left(\frac{M+\sqrt{M^{2}-Q^{2}}}{2 \sqrt{\theta}}\right)-\left(\frac{M+\sqrt{M^{2}-Q^{2}}}{\sqrt{\pi \theta}}\right) \exp \left(-\frac{\left(M+\sqrt{M^{2}-Q^{2}}\right)^{2}}{4 \theta}\right)\right], \\
& Q_{\theta_{+}}=Q \sqrt{\epsilon^{2}\left(\frac{M+\sqrt{M^{2}-Q^{2}}}{2 \sqrt{\theta}}\right)-\frac{\left(M+\sqrt{M^{2}-Q^{2}}\right)^{2}}{\sqrt{2 \pi \theta}} \exp \left(-\frac{\left(M+\sqrt{M^{2}-Q^{2}}\right)^{2}}{4 \theta}\right)},
\end{aligned}
$$

where $\epsilon(x)$ is the Gauss error function defined as

$$
\epsilon(x)=\frac{2}{\sqrt{\pi}} \int_{0}^{x} e^{-u^{2}} d u .
$$

As expected, for very large masses the function $\epsilon(x)$ tends to unity while the exponential term goes to zero, giving the classical $R N$ horizons $r_{\theta_{+}} \rightarrow r_{+}=M+\sqrt{M^{2}-Q^{2}}$.

\section{CONCLUSION}

In summary, we have investigated the tunneling of charged massive bosons $W^{ \pm}$from charged noncommutative $\mathrm{RN}$ black holes and BTZ black holes. The corresponding tunneling rates and the expected Hawking temperatures have been recovered by using the WKB approximation and the Hamilton-Jacobi equation in both cases. Furthermore, the results in this paper are consistent with an underlying unitary theory.

Last but not least, it is also shown that the remnants of the hawking temperature from the charged noncummutative black holes are increased by the effect of GUP. Therefore, this GUP-corrected Hawking radiation is common use for entire black holes. Then we have derived the logarithmic corrections in the leading order for the corrected entropy. Noted that the existence of the remnant depends critically on the full form of the entropy corrected by GUP. The corrected Hawking temperature and entropy are shown to depend on the noncommutaive parameter $\theta$ and quantum gravity effects $\alpha$. The black hole remnant would offer a way in which information might be preserved. Hence, the existence of a remnant may have important experimental consequences for the detection of black holes at the the Large Hadron Collider ( $\mathrm{LHC})$.

\section{ACKNOWLEDGMENT}

The authors would like to thank the anonymous reviewers.

[1] S. W. Hawking, Commun. Math. Phys. 43, 199 (1975); erratum-ibid, 46, 206 (1976).

[2] G. W. Gibbons and S. W. Hawking, Phys. Rev. D 15, 2738 (1977).

[3] I. Sakalli, M. Halilsoy, and H. Pasaoglu, Astrophys.Space Sci. 340, 155 (2012). 
[4] P. Kraus, F. Wilczek, Mod. Phys. Lett. A 9, 3713 (1994).

[5] P. Kraus, F. Wilczek, Nucl. Phys. B 437, 231 (1995).

[6] M.K. Parikh, F. Wilczek, Phys. Rev. Lett. 85, 5042 (2000).

[7] M.K. Parikh, Phys. Lett. B 546, 189 (2002).

[8] M.K. Parikh, Int. J. Mod. Phys. D 13, 2351 (2004).

[9] M. Angheben, M. Nadalini, L. Vanzo, S. Zerbini, J. High Energy Phys. 05, 014 (2005).

[10] K. Srinivasan, T. Padmanabhan, Phys. Rev. D 60, 024007 (1999).

[11] S. Shankaranarayanan, K. Srinivasan, T. Padmanabhan, Mod. Phys. Letts. 16, 571 (2001)

[12] J. Ahmed, K. Saifullah, JCAP 08, 011 (2011).

[13] K. Jusufi, Astrophys. Space Sci. 360, 22 (2015).

[14] L. Vanzo, G. Acquaviva, R. Di Criscienzo, Class. Quantum Gravity 28, 18 (2011).

[15] R. Kerner, R.B. Mann, Phys. Rev. D 73, 104010 (2006).

[16] R. Kerner and R.B. Mann, Class. Quant. Grav. 25, 095014 (2008).

[17] R. Kerner and R.B. Mann, Phys. Lett. B 665, 277-283 (2008).

[18] I. Sakalli, A. Ovgun, Astrophys Space Sci. 359, 32 (2015).

[19] A. Yale, R. B. Mann Phys. Lett. B 673, 168-172, (2009).

[20] S.I Kruglov, Mod. Phys. Lett. A 29, 1450203 (2014).

[21] S.I Kruglov, Int. J. Mod. Phys. A 29, 1450118 (2014).

[22] I. Sakalli, A. Ovgun, J.Exp.Theor.Phys, 121, 3, 404-407, (2015).

[23] I. Sakalli, A. Övgün, Gen.Rel.Grav. 48, 1 (2016).

[24] I. Sakalli, A. Ovgun, Eur. Phys. J. Plus 130: 110, (2015).

[25] G. Chen, Y. Huang, Int. J. Mod. Phys. A 30, 15, (2015).

[26] G. Chen, S. Zhou, Y. Huang, Astrophys Space Sci 357:51, (2015).

[27] A. Övgün, International Journal of Theoretical Physics, 1-9, (2016) , arXiv:1508.04100.

[28] P. Nicolini, A. Smailagic, E. Spallucci, Phys. Lett. B 632, 547, (2006).

[29] S. Ansoldi, P. Nicolini, A. Smailagic, E. Spallucci, Phys. Lett. B 645, 261-266, (2007).

[30] E. Spallucci, A. Smailagic, P. Nicolini, Phys. Lett. B 670 449-454, (2009).

[31] L. Modesto, P. Nicolini, Phys. Rev. D 82, 104035, (2010).

[32] A. Smailagic and E. Spallucci, J. Phys. A 36, L467 (2003).

[33] A. Smailagic and E. Spallucci, J. Phys. A 36, L517 (2003).

[34] A. Smailagic and E. Spallucci, J. Phys. A 37, 7169 (2004).

[35] S. Hamid Mehdipour, Int. J. Mod. Phys. A 25, 5543-5555, (2010).

[36] Y. Miao, Z. Xue, S. Zhang, Gen. Relativ. Gravit. 44 555-566, (2012).

[37] K. Nozari, S. H. Mehdipour, JHEP 0903, 061, (2009).

[38] K. Nozari, S. H. Mehdipour, Class. Quant. Grav. 25, 175015, (2008).

[39] M. Maggiore, Phys.Lett. B 304, 65-69 (1993)

[40] R. J. Adler, P. Chen, D. I. Santiago, Gen.Rel.Grav. 33, 2101-2108 (2001).

[41] A. Kempf, G. Mangano, R. B. Mann, Phys.Rev. D 52, 1108-1118 (1995).

[42] M. Faizal, M. M. Khalil, Int.J.Mod.Phys. A 30, no.22, 1550144, (2015).

[43] M.A. Anacleto, F.A. Brito, A.G. Cavalcanti, E. Passos, J. Spinelly, arXiv:1510.08444.

[44] X. Li, G. Chen, Physics Letters B 751 34-38, (2015).

[45] P. Nicolini, Int. J. Mod. Phys. A 24, 1229-1308, (2009).

[46] H. Kim, M. Park, C. Rim, J. H. Yee, JHEP 10 , 060, (2008) ; E.Chang -Young, D. Lee, Y. Lee, Class. Quant. Grav. 26, 185001, (2009).

[47] M. Banados, C. Teitelboim, J. Zanelli, Phys.Rev.Lett. 69, 1849-1851 (1992).

[48] M. Ammon, M. Gutperle, P. Kraus, E. Perlmutter, J.Phys. A 46, 214001 (2013).

[49] M. A. Anacleto, F. A. Brito, E. Passos, Phy. Lett. B 743, 184-188, (2015).

[50] J. Sadeghi and V.R. Shajiee, Int. J. Theor. Phys. 07, July (2015).

[51] S. H. Hendi, S. Panahiyan and R. Mamasani, Gen. Rel. Grav. 47, no. 8, 91 (2015).

[52] M. A. Anacleto, F . A. Brito, and E. Passos, Phys. Lett. B 749, 181 (2015).

[53] M. A. Anacleto, F . A. Brito, G. C. Luna, E. Passos, and J. Spinelly, Ann. Phys. 362, 436 (2015).

[54] A. F. Ali, S. Das, and E. C. Vagenas, Phys. Lett. B 678, 497 (2009).

[55] Y. Kahn and J. Thaler, Phys. Rev. D 86, 115012 (2012).

[56] V. A. Bednyakov, arXiv:1505.04380.

[57] J. Balewski, J. Bernauer, J. Bessuille, R. Corliss , R. Cowan, et al., arXiv:1412.4717.

[58] E. Gabrielli, L. Marzola, M. Raidal and H. Veermae, JHEP 1508, 150 (2015). 